\title{
Study of a progressive failure in soil using BEDS
}

\author{
Michael Iten ${ }^{1}$, Alexander M. Puzrin ${ }^{1}$, Dominik Hauswirth ${ }^{1}$, Stella Foaleng-Mafang ${ }^{2}$, Jean-Charles \\ Beugnot $^{2}$ and Luc Thévenaz ${ }^{2}$ \\ ${ }^{1}$ Institute for Geotechnical Engineering, ETHZ, CH-8093 Zurich, Switzerland \\ ${ }^{2}$ Institute of Electrical Engineering, EPFL, CH-1015, Lausanne, Switzerland
}

\begin{abstract}
In Geotechnical Engineering, progressive failure in soil-structure interaction is one of the least understood problems. It is difficult to study this phenomenon at laboratory scale, because of the large amount of strain gages required per unit length/area of the structure, which would interfere with the mechanical properties of both the structure and the soil. The recently developed Brillouin Echo Distributed Sensor (BEDS) technology overcomes this dilemma by distributed readings and $5 \mathrm{~cm}$ spatial resolution. A laboratory pullout testing program has been carried out to verify applicability of BEDS for the study of progressive failure in the soil-structure interaction.
\end{abstract}

Keywords: Soil-cable interaction, BEDS, Brillouin Echo Distributed Sensor, spatial resolution, optical fiber sensor, pullout box, laboratory sensor cable testing

\section{INTRODUCTION}

Progressive failure in soil-structure interaction is one of the most important and complicated problems in Geotechnical Engineering. So far it was not possible to study this phenomenon properly at laboratory scale, because of the large amount of strain gages required per unit length/area of the structure, which would interfere with the mechanical properties of both the structure and the soil. Optical fiber sensors provide an alternative, but have been so far limited to point sensors (Bragg Gratings) or distributed sensors with relatively large spatial resolution (BOTDA and others). The recently developed Brillouin Echo Distributed Sensor (BEDS) technology overcomes this dilemma by distributed readings and $5 \mathrm{~cm}$ spatial resolution. A laboratory testing program has been successfully carried out at the ETH Zurich to verify applicability of BEDS for the study of progressive failure in the soil-structure interaction.

\section{DISTRIBUTED FIBER SENSING SYSTEM}

The distributed fiber sensing system required a sub-metric spatial resolution an order of magnitude higher than a typical strain resolution obtained using traditional BOTDA systems. Clearly it was necessary to implement an advanced prototype configuration and the choice was oriented to a Brillouin Echo Distributed Sensor (BEDS) ${ }^{[1]}$ since it offers a flexibility and an operation principle equivalent to a classical BOTDA system, an essential quality for tests in a real environment.

The configuration that was set up to carry out the tests is the pump phase pulse technique, in which the pump wave is modulated by short $\pi$-phase pulses ${ }^{[2]}$. The pre-existing acoustic wave stimulated by the continuous pump and probe waves reflects the phase shifted pump and this tiny back-reflected out-of-phase light interferes destructively with the probe wave, resulting in an apparent loss proportional to the local amplitude of the pre-excited acoustic wave. The pulse duration being extremely short (500ps or $5 \mathrm{~cm}$ spatial resolution), the amplitude of the steady acoustic wave is so probed locally while showing all its natural narrowband characteristics in the spectral domain. This leads to a strain resolution of about $10 \mu \varepsilon$ (microstrains), that could be obtained by scanning the frequency of the probe wave by $1 \mathrm{MHz}$ steps.

The acquisition resolution was set to $25 \mathrm{ps}$ (equiv. to $0.25 \mathrm{~cm}$ ) to realize a good oversampling and to be only limited by the instrumental spatial resolution of $5 \mathrm{~cm}$. The data were then processed to extract the local central frequency of the Brillouin gain spectrum, requiring a special processing to eliminate the detrimental impact of the secondary echo on the measurements ${ }^{[2]}$.

20th International Conference on Optical Fibre Sensors, edited by Julian Jones, Brian Culshaw, Wolfgang Ecke, José Miguel López-Higuera, Reinhardt Willsch, Proc. of SPIE Vol. 7503, 75037S (c) 2009 SPIE · CCC code: 0277-786X/09/\$18 · doi: 10.1117/12.835115 
It must be pointed out that such an extreme spatial resolution requires a particularly cautious handling and positioning of the fiber since any bending of the optical cable induces a strain that is clearly observed in the distributed measured Brillouin spectrum. This fact is essential to interpret correctly the measured strain distributions, since very few sections show a uniform strain and the value of the zero-strain frequency is particularly hard to determine.

\section{LABORATORY TESTING SETUP}

\subsection{Pullout box}

For the laboratory testing, a $2 \mathrm{~m}$ long, $0.1 \mathrm{~m}$ wide and $0.2 \mathrm{~m}$ deep pullout box was used (Figure $1 \mathrm{a}$ ). The idea is that a structural part embedded in soil (e.g. an anchor or a cable buried at $0.1 \mathrm{~m}$ depth in sand) can be pulled out of the soil filled box. The pulling force is applied by a step motor at the front tip of the box. This motor allows for controlled pullout by displacement $\delta$. The pullout force $P$ is measured by a load cell.

This setup allows for various testing configurations. For the acquisition of the data presented in this paper, the following two tests were performed:

Free cable test: This setup is intended to obtain the stiffness (strain-stress dependency) for an optical cable section of length $\Delta L$. For this test, the box remains empty and a fiber cable is connected to a fixation point at the step motor and to another fixation point at a chosen distance $\Delta L$ from the step motor. The section $\Delta L$ can then be subjected to strain controlled by displacement $\delta$ of the step motor. At each strain step, optical measurements are carried out.

Buried cable test: In this test the cable is buried in sand and displacement $\delta$ is applied to it by the step motor. After chosen displacement step, optical measurements are carried out. The buried cable test allows for the specification of the distribution of shear stresses between the cable surface and the sand along the cable length. For softer cables this information is of crucial importance for understanding and modeling the progressive failure phenomenon.

\subsection{Fiber optic cables}

A custom produced specialty strain sensing fiber optic cable was used for the laboratory tests (Figure 1b). The cable is basically a well protected single mode fiber which shows very good strain transmission properties from the outer jacket to the fiber core. This cable has already been successfully applied for a landslide boundary evaluation in a field project ${ }^{[3]}$.

a)

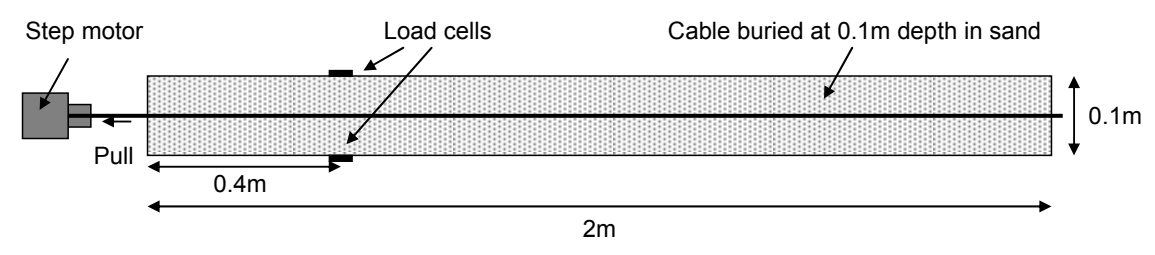

Fig. 1. (a) Pullout box; (b) Fiber optic cable crossection. b)

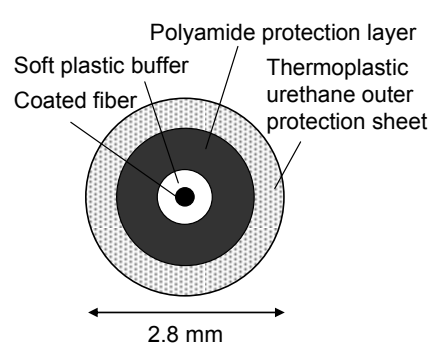

\section{LABORATORY TESTING RESULTS}

\subsection{Free cable testing}

Free cable testing was performed in a $2 \mathrm{~m}$ section of the cable. A comparison with similar testing using BOTDA technology showed an excellent correlation. Small fiber slippage inside the protection layer at relatively high strains $(>1.5 \%)$ was detected. Below that value, strain applied to the cable jacket was directly transferred to the fiber core. The data from the free cable tests was then used to back calculate stresses and forces from the optical measurements, which could be evaluated against the independently measured load cell forces. 


\subsection{Buried cable testing}

Buried cable testing was performed by pulling out a $2 \mathrm{~m}$ long cable section buried at $0.1 \mathrm{~m}$ depth in sand. Figure $2 \mathrm{a}$ shows the obtained strain data (relative to the zero strain measurement) along the fiber for eight displacement steps controlled by the step motor. The fixation of the cable to the step motor is at the distance of $2.83 \mathrm{~m}$ from the measuring unit. As is seen from Figure 2a, in the first four steps the strain behind the fixation point is zero. At larger displacement steps the strain profile propagates behind the fixation point, most probably due to the slippage of the glass fiber inside the cable. When converted into stresses (using the cable stiffness from the free cable tests), two distinctive portions of cable can be clearly identified for each step: in the first one the stress profile can be fitted by the straight line, in the second - by parabola (Figure 3b).

Validation of the optically measured strain against independent measurements is presented in Figure 3. First, numerical integration of the entire strain in the cable leads to the displacement at the fixation point $\delta$ and this can as well be compared with the value applied by the step motor (Figure 3a). Second, by using the optically measured maximum fiber strain at each load step, cable force at the fixation $P$ can then be calculated and compared to the applied load measured by the load cell (Figure $3 \mathrm{~b}$ ). As is seen, for both values $(P, \delta)$ the agreement between the optically and conventionally measured values is very good.
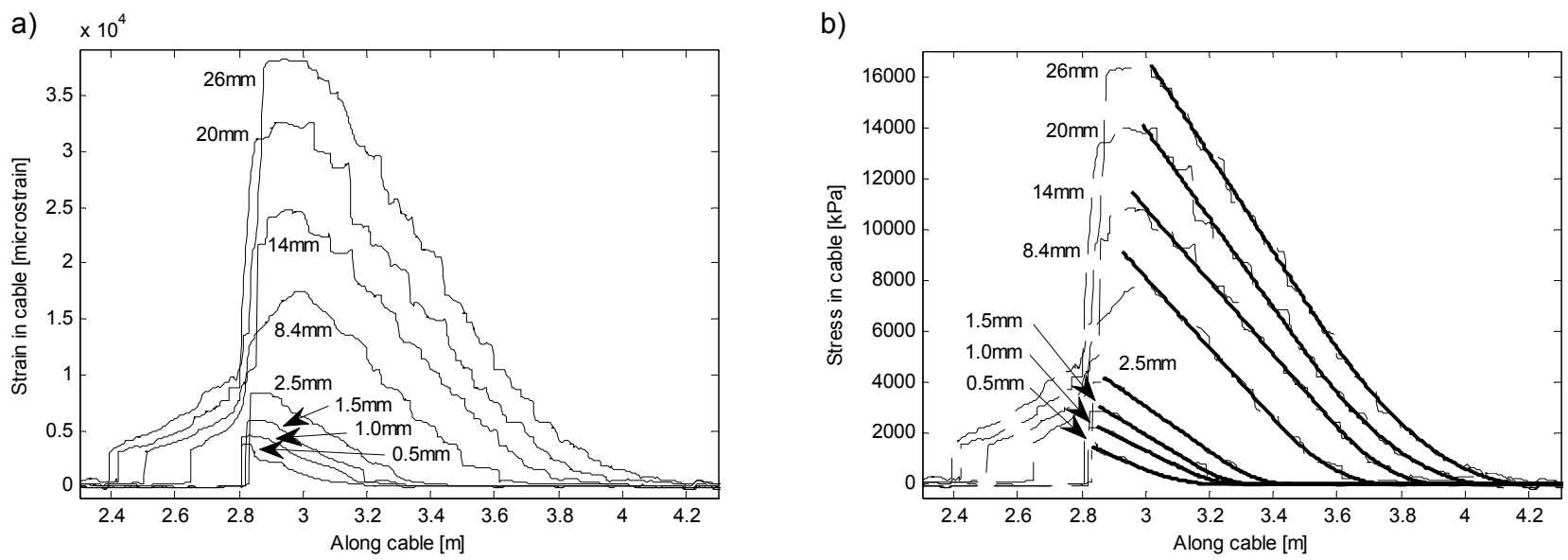

Fig. 2. (a) Optically measured strain for each load step $\delta$; (b) Calculated stress for each load step $\delta$ and fitted curves.

a)

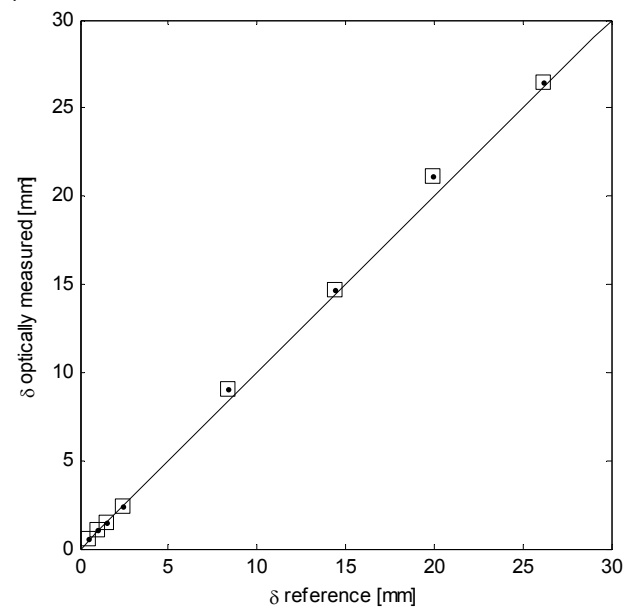

b)

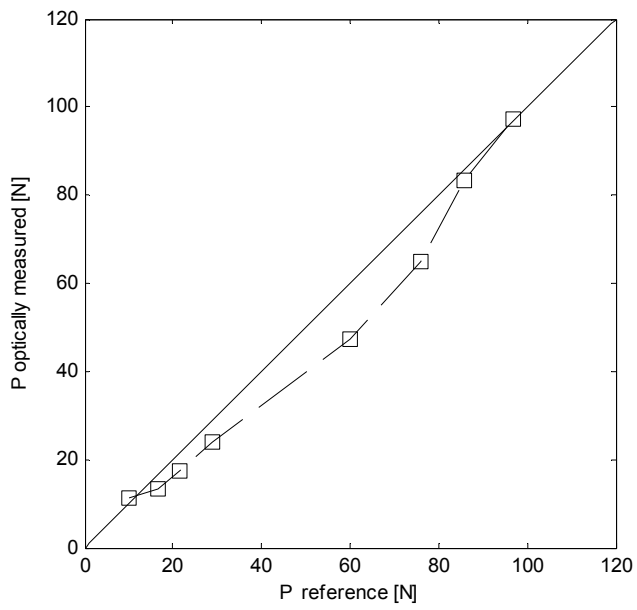

Fig. 3. (a) Applied vs. optically measured displacement; (b) Applied vs. optically measured pullout force. 


\section{GEOMECHANICAL INTERPRETATION}

When a cable is pulled out of sand, the soil fails at the contact with the cable. If the cable is sufficiently flexible, this failure does not occur simultaneously along the entire cable length, but rather initiates at the location of the maximum relative displacement (i.e., closest to the step motor) and propagates along the cable length with increased load. This type of failure is called progressive and its understanding and modeling is of high interest for geotechnical community. So far it was not possible to study this phenomenon properly at laboratory scale, because of the large amount of strain gages required, which would interfere with the mechanical properties of both the cable and the soil.

The novel BEDS technology provides an elegant solution to this problem. The cable is itself both the structure and the strain gage, allowing for at least 20 measuring points per meter, without affecting the stiffness of the cable or the soil. For the first time it became possible to observe the failure propagation at the lab scale with such a precision. In Figure 4a the shear stress (friction) acting on the cable is plotted against the cable length (obtained by differentiating the cable stress diagrams in Figure 2b). The portion where the failure has already taken place is represented by the constant shear stress, and, as expected, its length grows with the applied displacement. What is less obvious: (a) why this propagation slows down when the displacement increases (instead of accelerating and eventually leading to catastrophic failure), and (b) why the friction in this failure zone grows with the applied displacement (instead of decreasing to residual strength).

Answering these two questions would shed a new light into understanding of the progressive failure, and in fact, the authors have developed a model for the progressive failure in the buried cable test, which explains this phenomenon, but cannot be presented here due to the lack of space. One simple validation of this model, however, can be performed by plotting in Figure $4 \mathrm{~b}$ the optically measured stress in the cable at the end of the failure zone $\sigma_{L}$ (from Figure $2 \mathrm{~b}$ ) against the shear stress in this zone $\tau_{r}$ (from Figure $4 \mathrm{a}$ ). The model prediction is given by the formula:

$$
\sigma_{L}=a \cdot\left(\tau_{r}-\tau_{r 0}\right) \cdot \tau_{r}^{2}
$$

which is also plotted in Figure $4 \mathrm{~b}$, where it captures the trend of the data reasonably well. Identifying, understanding and modeling of these phenomena would not have been possible without the new data provided by the BEDS technology.

a)

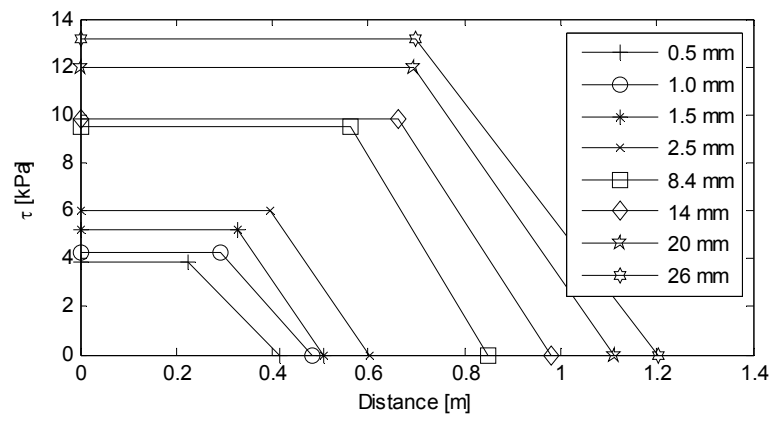

b)

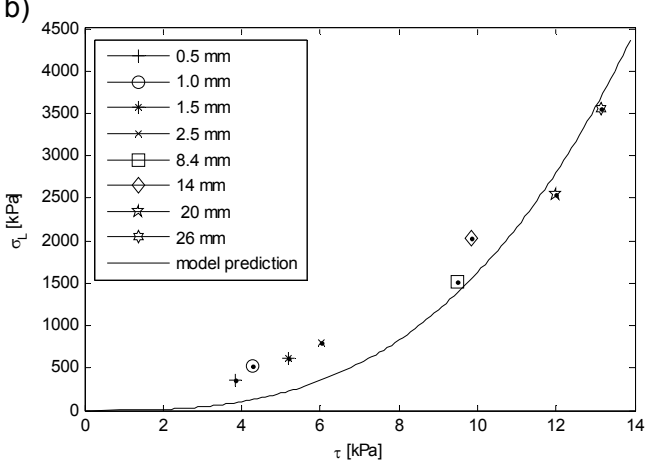

Fig. 4. (a) Shear stress distribution along the cable; (b) Stress in the cable at the end of the failure zone vs. the shear stress.

\section{ACKNOWLEDGEMENTS}

The authors would like to thank Omnisens for the support of the BEDS project, Brugg Cables for the strain sensor and Pascal Minder for his help during the lab campaign.

\section{REFERENCES}

[1] Thévenaz, L. and Beugnot, J.-C., "General analytical model for distributed Brillouin sensors with submeter spatial resolution", OFS-20 (2009 - in press).

[2] Foaleng Mafang, S., Beugnot, J.-C. and Thévenaz, L., "Optimized configuration for high resolution distributed sensing using Brillouin echoes", OFS-20 (2009 - in press).

[3] Iten, M., Schmid, A., Hauswirth, D. and Puzrin, A. M., "Defining and monitoring of landslide boundaries using fiber optic systems", Int. Symp. on Prediction and Simulation Methods for Geohazard Mitigation, Kyoto, Japan (2009). 